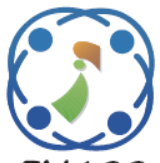

\title{
An Optimised Latent Fingerprint Matching System Using Cuckoo Search
}

\author{
Richa Jindal $^{1 *} \quad$ Sanjay Singla ${ }^{2}$ \\ ${ }^{1}$ Department of Computer Science and Engineering, IK Gujral Punjab Technical University, Jalandhar, India \\ ${ }^{2}$ Department of Computer Science and Engineering, Guru Gobind Singh College of Modern Technology, Kharar, \\ India \\ * Corresponding author’s Email: hrricha@gmail.com
}

\begin{abstract}
Biometrics is a scientific automation based on behavioural and physiological human traits to identify a person uniquely. Fingerprints are one of the eminent human psychological cues with an admiral potential for person identification. It has been widely adopted by many law enforcement agencies, forensics for criminal investigation. Amongst the three categories of fingerprints, latent fingerprints are the most intricate prints found in crime scenes and functions as dominant evidence in forensic investigations. The development of an accurate and reliable automatic latent fingerprint system is one of the pivotal issues for fingerprint researchers. This paper seeks the development of an optimal system for accurate latent fingerprint matching. The proposed system includes preprocessing of latent fingerprint image to remove noisy or unwanted information followed by minutiae extraction. The final step of proposed system includes the employment of swarm intelligence based cuckoo search algorithm to acquire optimal matching results. The performance of the system is evaluated on NIST Special Database 27 in terms of similarity score and identification rate. For the three categories of NIST dataset good, bad \& ugly, the proposed system has achieved similarity score value of $94.50 \%, 86.22 \% \& 84.78 \%$ and identification rates of $97.72 \%, 87.05 \%$ \& $83.52 \%$ respectively. The proposed system outperformed in comparison with other considered concept of latent fingerprint matching techniques.
\end{abstract}

Keywords: Latent fingerprint, Fingerprint recognition, Fingerprint enhancement, Biometric system, Swarm intelligence, Cuckoo search.

\section{Introduction}

In today's technological world, biometrics has earned a special place by replacing the traditional way of person identification. Biometric operates on behavioural and physiological cues like retina, iris, fingerprints, ear and facial thermo-gram etc. to authenticate a person. Fingerprint identification and matching is one of the most popular biometric processes. Many law enforcement agencies use fingerprint matching technology to identify criminal suspects [1]. Fingerprints are nothing but the impressions of fingertips consisting of line patterns. The line patterns are ridges and the spaces between the ridges are called as valleys [2]. The interleaved pattern of ridges and valleys is the most apparent characteristic of fingerprints [3].
The ridge structure of fingerprints is unique in nature. No two persons, not even twins have same fingerprints. Even injuries like cuts, superficial burns do not alter the ridge structure. When the new skin grows, the original ridge patterns are duplicated and thus making fingerprints so unique in nature [4]. The term minutiae are referred as the features of fingerprints at the local level. Minutiae are small details, the way in which ridge pattern is structured. Sometimes ridges terminate at some points, those points are referred as end points and sometimes these bifurcate which are termed as bifurcation point [5].

There are generally three types of fingerprints: rolled, plain and latent fingerprints as presented in fig. 1. Rolled fingerprints are obtained by rolling the finger "nail to nail" either on scanner or on a paper. Whereas, plain fingerprints are obtained either from 


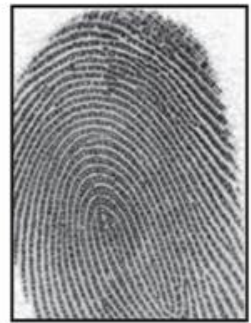

(a)

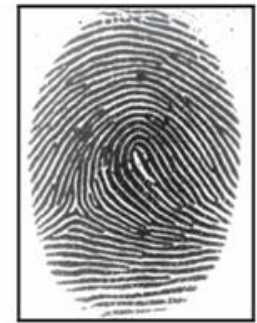

(b)

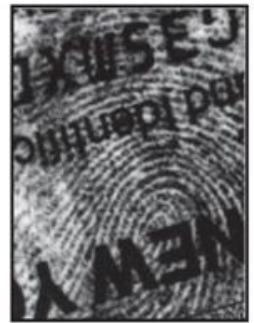

(c)
Figure. 1 Types of fingerprints: (a) rolled fingerprints, (b) plain fingerprints, (c) latent fingerprints

scanner or paper without rolling. Latent fingerprints are obtained from the surfaces which are touched accidently or inadvertently typically at a crime scene. Among the three types, latent fingerprints are the most usual and intricate evidence of person identification found at crime scene [6]. Latent fingerprints are complex in nature and are deposited when the fingertip consisting of natural secretions from sebaceous and eccrine gland comes in contact with any surface. Latent fingerprints are usually of poor quality due to presence of background noise and spoiled minutiae information as presented in fig. $1(\mathrm{c})$.

In forensics investigations, latent fingerprints hold utter importance to identify and convict criminals. In 1893, latent fingerprints were introduced for the first time as evidence in Argentina to convict a crime suspect [7]. This attracted numerous researchers to use various techniques to develop autonomous fingerprint recognition system. An "Automatic Fingerprint Identification System (AFIS)" was introduced for identification and matching of latent fingerprints. The goal of the AFIS was to provide a system for quick and automatic fingerprint matching. However, existing techniques are still not satisfactory in terms of speed and accuracy. The term "light out" was introduced to encourage the development of an autonomous latent fingerprint matching system without any human intervention. To inspire the researchers and to encourage the technological growth in the development of autonomous latent fingerprint matching system, FBI's CJIS (Criminal Justice Information Services) introduced "The Hit of the Year" award in 2007. The award symbolises the growth in the latent fingerprint matching technology. It also manifests the utility and advantage of latent fingerprint matching techniques in criminal investigations.

In order to develop an accurate and reliable AFIS, it is necessary to improve the quality of image before matching. Some techniques have been proposed to enhance the latent fingerprint image and to improve its quality [8-12]. Latent fingerprint image enhancement plays a key role in the process of fingerprint matching. In these methods, latent fingerprints are segmented and then enhanced to remove the structured noise and to improve the image quality respectively. An enhanced latent fingerprint image improves the possibility of prompt and precise fingerprint matching. The latent fingerprints are enhanced using the concept of orientation of the ridge structure and its estimation. The concept of estimation of orientation ridge structure has been studied extensively in literature [13-14]. A signal processing technique was proposed by Chikkerur et al. [13] for the fingerprint enhancement based on the estimation of ridge structure orientation. The authors proposed short time Fourier transform technique (STFT) to estimate the ridge orientation image, ridge frequency image and energy image to compute the region mask of the block of image. Wang et al. [14] proposed an orientation estimation model based on 2-D Fourier expansion named as FOMFE "Fingerprint Orientation Model based on 2-D Fourier Transform". The proposed model detects the singular points from the fingerprint region without any prior knowledge. Singular points are the points where the orientation of ridge terminates [15]. Two singular points: core and delta points are considered as registration points in the process of fingerprint matching [16-17]. Bazen and Gerez [16] proposed an algorithm to automatically detect the singular point as well as the directional filed of a pixel point. Yoon et al. [17] manually marked singular points for the orientation filed estimation. Further, Gabour filter was implemented for the fingerprint image enhancement. The method is simple and estimates the orientation near marked singular points, but it is poor in fingerprints like arch type having no singular points. Minutiae points are considered as local fingerprint features [18-19]. They consist of two ridge points: ridge ending and ridge bifurcation. The minutiae points are marked either manually or automatically to perform the task of latent fingerprint matching. The orientation field is estimated and then reconstructed based on minutiae information. In orientation filed estimation based models, filter is implemented to make the orientation fields smooth. But these methods have limitation in dealing with missing orientation patches and heavy noise value.

To refine the limitations of orientation field estimation approach, a dictionary based approach has been proposed [10-11]. The fingerprint orientations have different characteristics at different locations. A dictionary is considered based 
on the prior knowledge of fingerprint ridge structure. A reference orientation filed is estimated based on the orientation information stored in dictionary. The fingerprint image is initially decomposed using total variance decomposition model [10-11, 20]. The image is decomposed into two components: cartoon image component and texture image component. The structured noise is removed by eliminating the cartoon image component and the texture component containing the latent fingerprint information is considered for further processing. The dictionary is learnt from the prior information of orientation patches and reference orientation patch are constructed based on the dictionary information. Motivated by the recent advancements of dictionary based approach [10-11,20], this paper implements the dictionary based approach for latent fingerprint enhancement by reconstructing the dictionary based reference orientation patches and using Gaussian filter to make the smooth orientation field. Also, this paper aims to propose an optimal system for effective latent fingerprint matching. The objective of an optimal latent fingerprint matching system is achieved by implementing the system using swarm intelligence based cuckoo search optimization algorithm. In this paper, the latent fingerprint image is initially pre-processed by following the steps of segmentation, enhancement and binarization for effective latent fingerprint feature extraction. The local level latent fingerprint features i.e. minutiae points are extracted automatically and then cuckoo search optimization algorithm is implemented for latent fingerprint matching.

The rest of the paper is outlined as follows. The paper is enclosed with total number of seven sections. Section 2 includes the pre-processing techniques for latent fingerprint image. Section 3 and 4 describes the minutiae based feature extraction and removal of false minutiae respectively. Section 5 includes the discussion of cuckoo search optimization algorithm for minutiae matching followed by section 6 presenting experimental and comparison results. At last, section 7 concludes the paper.

\section{Latent fingerprint pre-processing}

Latent fingerprint pre-processing involves upgrading the latent fingerprint image to reduce or to eliminate the possibility of false feature extraction. Latent fingerprint images are of very poor quality. The images are generally collected from crime scenes and consist of noise data e.g. textual data as presented in image 1(c). The latent fingerprint images need to be processed first so that necessary information could be easily extracted from the fingerprint image. The pre-processing phase is similar to preparing the data for further processing. The four processes considered to pre-process the latent fingerprint image data are Latent fingerprint Capture, Latent fingerprint Segmentation, Latent fingerprint Enhancement, Image Binarization.

\subsection{Latent fingerprint capture}

The primary step in the process of latent fingerprint detection is to capture the latent fingerprints. Latent fingerprints are impressions which are left accidently at crime scenes. The crime scene investigators collect/capture the fingerprints at crime scenes using various techniques of physical and chemical science. The selection of technique entirely depends on the geometry and material of the surface from which the fingerprint is lifted. The techniques include UV based, solvent based, powder based, fuming based, contact-less lifting and electromagnetic based fingerprint lifting [21]. In this paper, experimentation is performed on NIST SD27 [22] expert latent fingerprint dataset. NIST SD27 is a greyscale latent fingerprint special database collected by National Institute of Standard and Technology in union with Federal Bureau of Investigation. The dataset consists of a total number of 258 latent fingerprint cases collected from crime scenes along with their matching fingerprint mates. Fig. 2 presents the sample latent fingerprint images from NIST SD 27 dataset.

\subsection{Latent fingerprint segmentation}

Latent fingerprint segmentation is the first step in the process of latent fingerprint matching. Segmentation includes separating the foreground image containing useful latent fingerprints to the unwanted background noises. The task of segmentation is demanding and challenging because it involves estimation of the useful and relevant foreground boundary. Segmentation is the process of extracting useful information and eliminating the un-necessary background noise. The objective of this step is to mark the region containing the fingerprint information accurately. The region containing the useful fingerprint information is called as Region of Interest (ROI). In the proposed latent fingerprint matching system the steps considered for the latent fingerprint image segmentation are: 


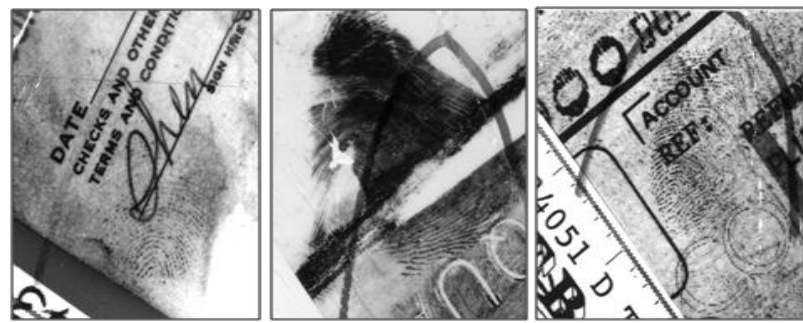

Figure. 2 Sample latent fingerprint images from NIST SD27 dataset

- The latent fingerprint image is initially segmented into number of blocks with each block of size $16 \times 16$ pixels.

- The variance for each block of pixels is computed and based on the computed variance, a threshold value is specified

- The pixel blocks with variance less than the specified threshold value are eliminated and remaining blocks are considered for further processing.

\subsection{Latent fingerprint enhancement}

Before feature extraction, the process of latent fingerprint enhancement is absolute essential and important. Latent fingerprint enhancement improves the quality of the latent fingerprint image. Enhancement supports feature extraction process by improving the quality of ridge structure of latent fingerprint. In this paper, a dictionary based approach is introduced for the enhancement of the latent fingerprint image. Dictionary is constructed with prior information of the ridge structure with different orientation patterns. In order to make robust and reliable dictionary, high quality rolled fingerprint patches are selected. The orientation field is estimated from the segmented latent fingerprint images. Latent fingerprints have unclear ridge structure, so dictionary is used to reconstruct the estimated orientation of ridge structure. Further, Gaussian filter is used to enhance the latent fingerprint image. Gaussian filter is a linear filter used to effectively remove the Gaussian noise in order to smooth the image. The filter is computationally efficient and also eliminates image blurring. The weights in Gaussian filter are selected according to the Gaussian functions. The degree of smoothening depends on the value of $\sigma$. Larger the value of $\sigma$, more intense will be smoothening of the fingerprint image. A two variable Gaussian function is formulated as presented in Eq. (1).

$$
g(x, y)=\left[\frac{1}{\sqrt{2 \pi \sigma}} e \frac{x^{2}}{2 \sigma^{2}}\right] \times\left[\frac{1}{\sqrt{2 \pi \sigma}} \frac{y^{2}}{e 2 \sigma^{2}}\right]
$$

Gaussian filter is applied on the latent fingerprint image to enhance the image quality and to remove the noise present in the image. The high boost Gaussian filter is applied with amplification factor to improve the image clarity. Finally, an enhanced image with better quality is obtained after this process.

\subsection{Image binarization}

Image binarization is the process of transforming the gray scale latent fingerprint image into binary image. The gray scale latent fingerprint image with pixel value upto 256 is converted into binary image with pixel value either 0 or 1 . For the process of image binarization, a threshold value is evaluated and all the pixels are classified according to the specified threshold value. The pixels with value above than threshold are classified as pixels with value 1 and rest as pixels with 0 values. The process of local adaptive binarization is performed to transform the gray scale latent fingerprint image into binary image. The image is divided into number of blocks, each of size $16 \times 16$ pixels. The threshold value is evaluated by calculating the mean intensity value of each block of pixels. The pixel value in the block is transformed to 1 if its value is greater than the evaluated mean intensity value of the block.

\section{Latent fingerprint feature extraction}

Features are the most crisp and succinct representation of data in any form. The success of latent fingerprint matching system lies in the selection and extraction of robust features. Fingerprints are unique in nature, so the features representing the fingerprint data should maintain its property of uniqueness. Due to poor ridge information, the task of feature extraction from latent fingerprints is very challenging. Fingerprint features are broadly categorized into three levels. Level 1 features consist of overall ridge flow pattern, level 2 features are the minutiae points and level 3 features are extended features including information about pores and dots etc.

In the proposed latent fingerprint matching system, level 2 features are extracted for fingerprint matching. Minutiae points are local fingerprint features consisting of ridge ending and ridge bifurcation points. The minutiae points are extracted automatically by performing a local pixel analysis. The binarized latent fingerprint image obtained after pre-processing is scanned both vertically and horizontally using a pixel window of size $3 \times 3$. The method computes the number of 1's for each pixel window and classifies the pixel either into normal, 
ridge ending point or ridge bifurcate point. Fig. 3 presents the classification of the pixel in a $3 \times 3$ window into ridge ending, ridge bifurcation and normal pixel respectively. The rules for pixel point classification are:

- If the central pixel in the $3 \times 3$ pixel window is 1 and there is only one pixel with value 1 in the neighbouring pixels, then the central pixel is a ridge ending point.

- If the central pixel in the $3 \times 3$ pixel window is 1 and there are three pixels with value 1 in the neighbouring pixels, then the central pixel represents point with ridge bifurcation.

- If the central pixel in the $3 \times 3$ pixel window is 1 and there are two pixels with value 1 in the neighbouring pixels, then the central pixel is a normal pixel.

\section{Removing spurious or false minutiae}

After the pre-processing of the latent fingerprint image, minutiae are extracted. There is always a possibility of occurrence of extraction of false minutiae during the extraction. The presence of false or spurious minutiae decreases the overall performance of a latent fingerprint matching system which makes their removal essential. The process of removing false minutiae is a post processing technique performed after the minutiae extraction.

\begin{tabular}{|c|c|c|}
\hline 1 & 0 & 0 \\
\hline 0 & 1 & 0 \\
\hline 0 & 0 & 0 \\
\hline
\end{tabular}

(a)

\begin{tabular}{|l|l|l|}
\hline 1 & 0 & 0 \\
\hline 0 & 1 & 0 \\
\hline 1 & 0 & 1 \\
\hline
\end{tabular}

(b)

\begin{tabular}{|l|l|l|}
\hline 1 & 0 & 0 \\
\hline 0 & 1 & 0 \\
\hline 0 & 0 & 1 \\
\hline
\end{tabular}

(c)
Figure. 3 Classification of the pixel: (a) ridge ending (b) ridge bifurcation (c) normal pixel

Table 1. Examples of false minutiae

\begin{tabular}{|l|l|l|}
\hline & Break & \\
\hline
\end{tabular}

False minutiae include ridge structures in the form of triangle, spike, spur, break, island etc [23]. Table 1 presents the examples of false minutiae.

To remove the false minutiae, a threshold value $R$ is specified. Let $S_{m}$ defines the sum of pixels in a row with value 1 and $L_{r}$ defines the length of row. Then, the average distance $\left(\mathrm{AV}_{\mathrm{d}}\right)$ between the two neighbouring ridge pixel points is calculated using Eq. (2).

$$
A V_{d}=\frac{S_{m}}{L_{r}}
$$

The procedure to detect and remove the false minutiae points is based three rules. The rules are:

- If the distance between two ridge ending points is less than $R$ and both points are located on same ridge, then remove both the ridge ending points.

- If the distance between two ridge bifurcation points is less than $R$ and both points are located on same ridge, then remove both the ridge bifurcation points.

- If the distance between the ridge ending and bifurcation point is less than $R$ and both points are located on same ridge, then remove both the minutiae points.

\section{Minutiae matching by cuckoo search}

After the minutiae extraction, the last step in the process of proposed latent fingerprint matching system is the minutiae matching. The proposed latent fingerprint matching system aims to find the optimal match of the input latent fingerprint image from the rolled fingerprint repository. In order to find the optimal match, swarm intelligence based cuckoo search optimization algorithm is implemented. The flowchart of the proposed latent fingerprint matching system is presented in fig. 4. In this section the proposed minutiae matching algorithm using cuckoo search is discussed.

Cuckoo search [24-26] optimization algorithm was proposed by carefully perusing the behavioural characteristics of different species of cuckoo bird. The beautiful sound that cuckoo birds produce is extremely enchanting and alluring. At the same time, these birds are immensely captivating due to their behavioural aspect of laying their own eggs in the nest of host bird. Cuckoo birds are characterized as brood parasites because they rely on other birds to raise their young. Many cuckoo bird species like ani and guira remove the host bird's eggs from their nests and lay their own eggs to increase their hatching probability [24]. In case host birds 
recognize the external egg in their nests, the situation leads to two possibilities. The host bird will either throw the cuckoo bird's egg or will abandon its own nest and build a new nest elsewhere. Some cuckoo bird species like tapera have developed an ability to imitate the colour and the pattern of the eggs of selected host bird species [27]. Also the propensity of cuckoo birds in terms of selecting of host bird's nest and timing of laying eggs is remarkable. Host bird who has just laid eggs is picked by cuckoo bird using the levy flight property. Levy flight [28] property allows random walks based on probability density function. Levy flight is used by cuckoo bird to generate nest at each iteration. The position of nest is selected randomly to levy flight. The egg will be place by comparing the values of objective function and thereby ensuring the optimal nest for cuckoo bird's egg. Yang and Deb [24] specified three rules to simplify the cuckoo search optimization algorithm. The rules are listed as:

- Cuckoo bird will lay only one egg at a time and dump it at host bird's nest selected randomly.

- The best nests carrying high quality eggs will carry forward the nest generation.

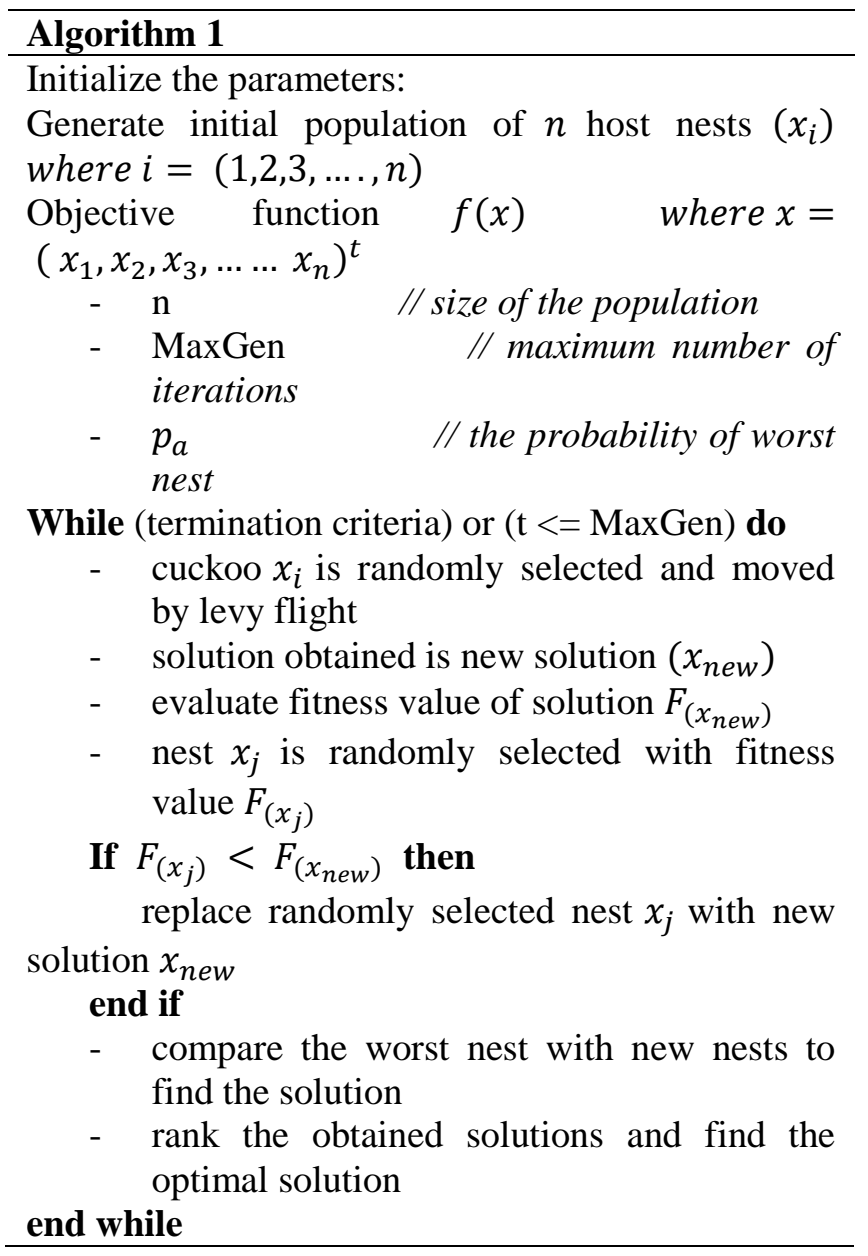

- The number of available host nests is fixed and $p_{a} \in[0,1]$ is defined as the probability of discovery of external egg of cuckoo bird by host.

In case of rule 3, two possibilities are there. The host bird will either throw the cuckoo bird's egg or will abandon its own nest and build a new nest elsewhere.

Algorithm 1 illustrates the process of minutiae matching using cuckoo search is proposed. The two minutiae points: ridge ending and ridge bifurcation are extracted as fingerprint features. The minutiae points are considered as pattern points. Thus the problem of minutiae matching can be considered as pattern point matching problem. The brood parasitic cuckoo bird's pattern mimicking property is used to match the minutiae points and Levy flight is used by cuckoo bird to generate nest at each iteration in order to propose new solution. If $x(t)$ is the current solution, then the new solution $x(t+1)$ for $\mathrm{i}^{\text {th }}$ cuckoo can be generated by using Eq. (3).

$$
x_{i}(t+1)=x_{i}(t)+\alpha \oplus \operatorname{Levy}(\lambda)
$$

Here $\alpha$ is scaling factor for random walk by levy flight. The new solution depends on the current solution and the probability $p_{a}$

\section{Experimental results}

The performance of the system is evaluated in terms of similarity score and identification rate. Two experiments are performed in terms of aforementioned parameters for the performance evaluation of the proposed automated latent fingerprint matching system. The first experiment involves the analysis and comparison of the similarity score of proposed system with results achieved by Kumar \& Velusamy [29]. In the second experiment, system's identification rate is compared with results assessed by Venkatesh et al. [31]. The detailed description of the two experiments is discussed in the following subsections.

In the proposed work, experimentation is performed on NIST Special Database 27 (SD-27). NIST SD-27 is a latent fingerprint image dataset that is widely used for the experimental evaluation of many latent fingerprint applications including enhancement, identification and matching. The dataset is collected from crime scenes which is the reason for its low quality fingerprint images. The dataset includes 258 images of both latent and their corresponding rolled fingerprint images. The 258 latent fingerprint images are further classified into three categories based on the quality of the image 


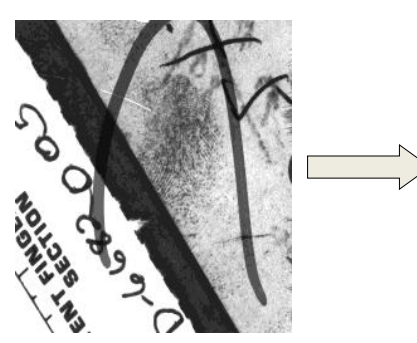

(a)

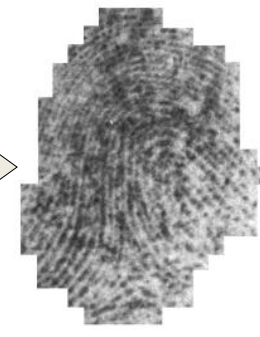

(b)

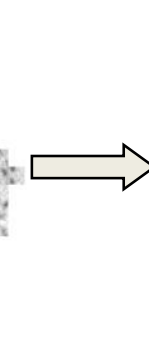

(c)

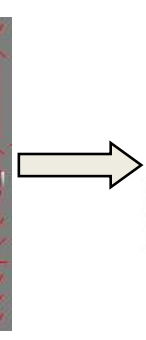

c)

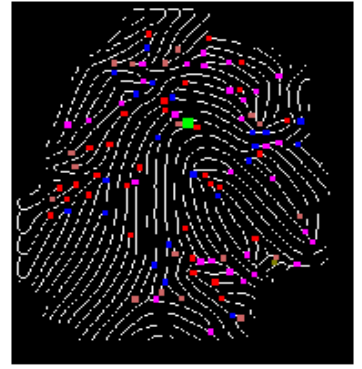

(f)
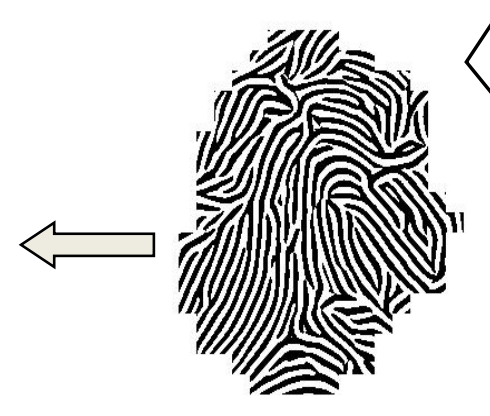

(e)

(g)

Figure. 4 Flowchart of proposed latent fingerprint matching system: (a) original latent fingerprint image (b) segmented latent fingerprint image $(c)$ enhanced latent fingerprint image with reconstructed orientation using dictionary (d) enhanced latent fingerprint image (e) binarized latent fingerprint image (f) automatically marked minutiae points (g) extracted minutiae points

which are: Good, bad and Ugly. The Good latent fingerprint image category includes 88 images and the ad \& Ugly categories contain 85 images each.

The experiments are implemented on MATLAB version 8.3.0.532 running on system with configuration follows: Processor- Intel(R) Core(TM) i5@ $3.20 \mathrm{GHz}, \mathrm{RAM}-4.00 \mathrm{~GB}$ and System type64-bit.

\subsection{Experiment I}

Experiment I includes the results subject to similarity score measure of latent fingerprint images. The similarity score refers to the calculation of minutiae similarity of the latent fingerprint image with respect to the rolled images present in the database. Let $M^{L}$ refers to the set of minutiae of the latent fingerprint image and $M^{D}$ is the set of minutiae of the rolled fingerprint image from database as shown in Eq. (4) and Eq. (5).

$$
\begin{aligned}
& M^{L}=\left(x^{l}, y^{l}, \alpha^{l}\right) \\
& M^{D}=\left(x^{D}, y^{D}, \alpha^{D}\right)
\end{aligned}
$$

The variables $\left(x^{l}, y^{l}\right),\left(x^{D}, y^{D}\right)$ and $\alpha^{l}, \alpha^{D}$ denotes the location and orientation of two minutiae sets respectively. During the experimentation, the similarity score of every latent fingerprint image is calculated corresponding to the rolled fingerprint images in the database. The proposed system has achieved an overall similarity score of $88.50 \%$. The latent fingerprint image dataset is classified into three categories Good, Bad and Ugly based on the quality of the image. The similarity score corresponding to the aforementioned three categories is $94.50 \%, 86.22 \%$ and $84.78 \%$ respectively. The graphical representation of the overall system's result is presented in fig. 5 .

For further evaluation of the proposed system in terms of similarity score, another experiment similar to the experiment conducted by Kumar \& Velusamy

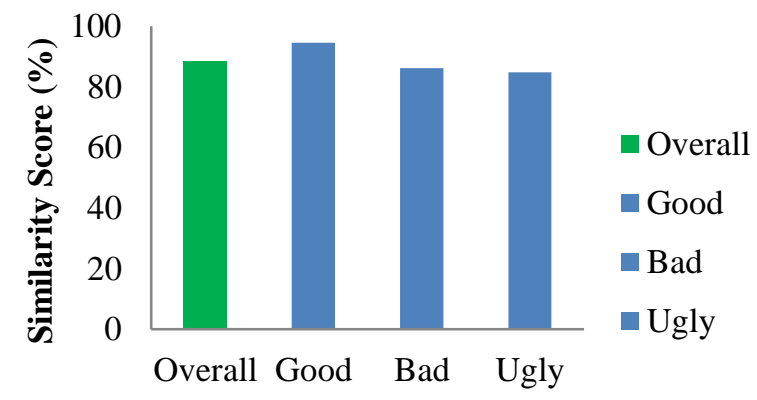

Latent Fingerprint Category

Figure. 5 The overall similarity score result of proposed system 
[29] is performed. Kumar \& Velusamy calculated the average similarity score and compared the results with [30] observing an increase in average similarity score by $1.6 \%$. The same experiment when performed with proposed system has evinced an increase in average similarity score by $8.56 \%$.

\subsection{Experiment II}

Experiment II includes the results subject to the identification rate of latent fingerprint images. Latent fingerprint matching is a one-to-many matching problem which can be further viewed as a series of one-to-one matching problem. In the experimentation, every latent fingerprint is matched with every rolled fingerprint image stored in database. During the experimentation, the ground truth values are considered as benchmark for the selection of accurate identified match. The fingerprint image with minutiae similarity greater than or equal to $75 \%$ (threshold value) with respect to ground truth value is nominated as the identified match (ground truth match). The identification rate of the system is the ratio of number of identified matches to the total number of images as presented in Eq. (6).

$$
\text { Identification rate }=\frac{\text { number of identified matches }}{\text { total number of images }}
$$

The quality of the fingerprint image directly influences by the identification rate. Separate experiments are performed for the Good quality latent fingerprint images followed by Bad and Ugly latent fingerprint images. The overall identification rate achieved by Good, Bad and Ugly quality fingerprint image is $97.72 \%, 87.05 \%$ and $83.52 \%$ respectively.

The performance of the proposed system is further evaluated by comparing the results evaluated by Venkatesh et al. [31]. In this comparison, results

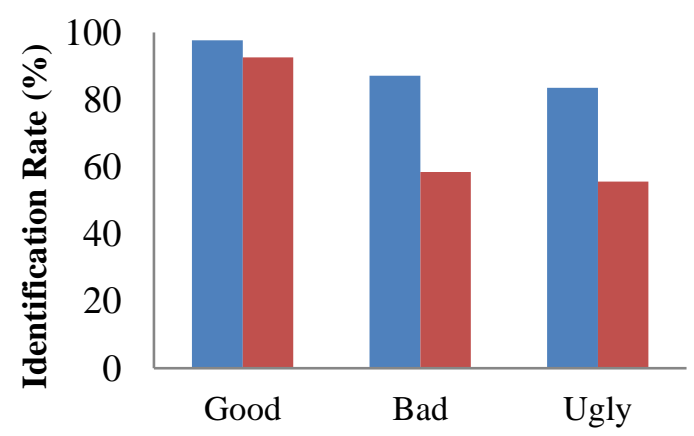

Latent Fingerprint Catergory

Figure. 6 Comparison of proposed system with Venkatesh et al. [31] on the basis of identification rate
Table 1. Comparative results of proposed system with Venkatesh et al. [31]

\begin{tabular}{|l|c|c|c|}
\hline \multirow{2}{*}{\multicolumn{1}{|c|}{ Techniques }} & \multicolumn{3}{|c|}{ Identification Rate (\%) } \\
\cline { 2 - 4 } & Good & Bad & Ugly \\
\hline Proposed System & 97.72 & 87.05 & 83.52 \\
\hline Venkatesh et al. [31] & 92.6 & 58.5 & 55.6 \\
\hline
\end{tabular}

evaluated by Venkatesh et al. [31] using neuro fuzzy system with NIST SD-27 are considered. Fuzzy system has the ability to handle the vagueness and uncertainty of fingerprints. But the combination of their membership functions is not robust that make it difficult to implement and evaluate the accurate results. On the other hand, Cuckoo Search works on brood parasitism property having ability to handle large data values with ease. The major properties responsible for this simplicity are usability of lesser parameters (only probability distribution and population size) and ongoing learning behaviour with iterations. These properties make cuckoo search easy to implement in comparison with other concepts. The experimental evaluated results performed on NIST SD-27 also show the superiority of the cuckoo search concept. In this evaluation, NIST SD-27 results are categorised in terms of quality of latent fingerprint image. Table 1 and fig. 6 present the comparative results of proposed system with Venkatesh et al. [31].

The identification results of Good quality latent fingerprint images have achieved maximum identification rate of $97.72 \%$. This means that out of 88 Good quality latent fingerprint images 86 image are correctly identified. An identification rate of $87.05 \%$ and $83.52 \%$ corresponding to Bad and Ugly quality latent fingerprint images indicate that out of 85 images 74 and 71 images are correctly identified respectively. On the other hand, the results evaluated by Venkatesh et al. [31] lack the system performance as shown in fig. 6. The results evaluated by proposed system indicate the higher value of identification rate.

\section{Conclusion}

In this paper, swarm intelligence based cuckoo search optimization algorithm is proposed for the autonomous latent fingerprint matching system. The problem of latent fingerprint minutiae matching is considered as pattern point matching problem and the brood parasitic pattern mimicking property of cuckoo bird is used as solution for minutiae matching. This paper seeks the development of system providing optimal matching result. The development of the system is followed by several modules and sub-modules. Initially, the possibility to extract false minutiae information is eliminated 
by pre-processing the image dataset. Pre-processing module can be viewed as data preparation module for further processing. The module includes latent fingerprint capture, latent fingerprint segmentation, latent fingerprint enhancement and image binarization as its sub-modules. The system proceeds with the extraction of level 2 features (minutiae) by following a local-pixel analysis. The integrity of the extracted features is maintained by specifying rules for the elimination of false minutiae. Finally cuckoo search algorithm is employed for optimal minutiae matching.

The performance of the proposed system is evaluated in terms of similarity score and identification rate. The experiments are conducted using NIST SD-27 latent fingerprint image dataset which is categorised into Good, Bad and Ugly categories based on image quality. The proposed system has achieved an overall similarity score of $88.50 \%$ and a similarity score value of $94.50 \%$, $86.22 \%$ and $84.78 \%$ for the aforementioned dataset categories. The identification rates achieved by the system with respect to the three preceding dataset categories are $97.72 \%, 87.05 \%$ and $83.52 \%$. In addition, the performance of the proposed system is further evaluated by comparing the results with existing technique. The proposed system has evinced significant comparative results.

For future reference, the proposed system for latent fingerprint recognition can be integrated with other meta-heuristic approach having property of global optimization with the use of multiple agents. This may further enhance the recognition rate for the bad and ugly fingerprints.

\section{References}

[1] C. Champod, C. J. Lennard, P. Margot, and M. Stoilovic, Fingerprints and other ridge skin impressions, CRC press, 2016.

[2] A. K. Jain, S. Prabhakar, L. Hong, and S. Pankanti, "Filterbank-based fingerprint matching", IEEE Transactions on Image Processing, Vol. 9, No. 5, pp.846-859, 2000.

[3] S. S. Omran and M. A. Salih, "Design and Implementation of Multi-Model Biometric Identification System", International Journal of Computer Applications, Vol. 99, No. 15, pp.1421, 2014.

[4] D. Maltoni, D. Maio, A. Jain, and S. Prabhakar, Handbook of fingerprint recognition. Springer Science \& Business Media, 2009.

[5] N. Ratha, and R. Bolle (Eds.), Automatic fingerprint recognition systems, Springer Science \& Business Media, 2007.
[6] M. Zhang, X. Yu, G. Qin, Y. Zhu, M. Wang, Q. Wei, Y. Zhang, and X. Zhang, "Latent fingerprint enhancement on conductive substrates using electrodeposition of copper", Science China Chemistry, Vol. 58, No. 7, pp.1200-1205, 2015.

[7] M. Hawthorne, Fingerprints: analysis and understanding, CRC Press, 2008.

[8] S. Yoon, J. Feng, and A. K. Jain, "Latent fingerprint enhancement via robust orientation field estimation", In: Proc. of 2011 International Joint Conference on Biometrics (IJCB), pp.1-8, 2011.

[9] J. Zhang, R. Lai, and C. J. Kuo, "Latent fingerprint segmentation with adaptive total variation model", In: Proc. of 2012 5th IAPR International Conference on Biometrics (ICB), pp.189-195, 2012.

[10] J. Feng, J. Zhou, and A. K. Jain, "Orientation field estimation for latent fingerprint enhancement", IEEE Transactions on Pattern Analysis and Machine Intelligence, Vol. 35, No. 4, pp.925-940, 2013.

[11] K. Cao, E. Liu, and A. K. Jain, "Segmentation and enhancement of latent fingerprints: A coarse to fine ridge structure dictionary", IEEE Transactions on Pattern Analysis and Machine Intelligence, Vol. 36, No. 9, pp.1847-1859, 2014.

[12] X. Yang, J. Feng, and J. Zhou, "Localized dictionaries based orientation field estimation for latent fingerprints", IEEE Transactions on Pattern Analysis and Machine Intelligence, Vol. 36, No. 5, pp. 955-969, 2014.

[13] S. Chikkerur, A. N. Cartwright, and V. Govindaraju, "Fingerprint enhancement using STFT analysis", Pattern Recognition, Vol. 40, No. 1, pp.198-211, 2007.

[14] Y. Wang, J. Hu, and D. Phillips, "A fingerprint orientation model based on 2D Fourier expansion (FOMFE) and its application to singular-point detection and fingerprint indexing", IEEE Transactions on Pattern Analysis and Machine Intelligence, Vol. 29, No. 4, pp.573-585, 2007.

[15] V. S. Srinivasan and N. N. Murthy, "Detection of singular points in fingerprint images", Pattern Recognition, Vol. 25, No. 2, pp.139-153, 1992.

[16] A. M. Bazen and S. H. Gerez, "Systematic methods for the computation of the directional fields and singular points of fingerprints", IEEE Transactions on Pattern Analysis and Machine Intelligence, Vol. 24, No. 7, pp.905-919, 2002. 
[17] S. Yoon, J. Feng, and A. K. Jain, "On latent fingerprint enhancement", In: Proc. of Biometric Technology for Human Identification VII, Vol. 7667, pp.228, 2010.

[18] A. A. Paulino, A. K. Jain, and J. Feng, "Latent fingerprint matching: Fusion of manually marked and derived minutiae", In: Proc. of 2010 23rd SIBGRAPI Conference on Graphics, Patterns and Images (SIBGRAPI), pp.63-70, 2010.

[19] V. Jain and A. K. Singh, "An approach for Minutia Extraction in Latent Fingerprint Matching", International Journal of Innovations in Engineering and Technology, Vol. 6, No. 1, pp. 51-58, 2015.

[20] M. Liu, X. Chen, and X. Wang, "Latent fingerprint enhancement via multi-scale patch based sparse representation", IEEE Transactions on Information Forensics and Security, Vol. 10, No. 1, pp.6-15, 2015.

[21] T. Kent and U. K. Lincoln, "Visualization or development of crime scene fingerprints", Forensic Fingerprints, pp.43-58, 2016.

[22] M. D. Garris and R. M. McCabe, "Fingerprint minutiae from latent and matching tenprint images", National Institute of Standards and Technology, pp.1-36, 2000.

[23] F. Zhao and X. Tang, "Preprocessing and postprocessing for skeleton-based fingerprint minutiae extraction", Pattern Recognition, Vol. 40, No. 4, pp. 1270-1281, 2007.

[24] X. S. Yang and S. Deb, "Cuckoo search via Lévy flights", In: Proc. of World Congress on
Nature \& Biologically Inspired Computing, pp.210-214, 2009.

[25] X. S. Yang and S. Deb, "Engineering optimisation by cuckoo search", International Journal of Mathematical Modelling and Numerical Optimisation Vol. 1, No. 4, pp.330343, 2010.

[26] X. S. Yang, and S. Deb, "Multiobjective cuckoo search for design optimization", Computers \& Operations Research, Vol. 40, No. 6, pp.1616-1624, 2013.

[27] R. B. Payne and M. D. Sorensen, The cuckoos, Vol. 15, Oxford University Press, 2005.

[28] I. Pavlyukevich, "Lévy flights, non-local search and simulated annealing", Journal of Computational Physics, Vol.226, No. 2, pp.1830-1844, 2007.

[29] S. Kumar and R. L. Velusamy, "Kernel approach for similarity measure in latent fingerprint recognition", In: Proc. of International Conference on Emerging Trends in Electrical Electronics \& Sustainable Energy Systems (ICETEESES), pp.368-373, 2016.

[30] URL:

http://www.mathworks.com/matlabcentral/filee xchange/44369-fingerprint-matching--asimple-approach.

[31] R. Venkatesh, N. U. Maheswari, and S. Jeyanthi, "Multiple Criteria Decision Analysis Based Overlapped Latent Fingerprint Recognition System Using fuzzy Sets", International Journal of Fuzzy Systems, Vol. 20, No.5, pp. 1-27, 2018. 